\title{
Combined proton and carbon ion radiotherapy for uterine cervical squamous cell carcinoma: A retrospective analysis
}

Zhan Yu ( $\sim$ yuzhanecho@foxmail.com )

Shanghai Proton and Heavy Ion Center

\section{Zhengshan Hong}

Shanghai proton and Heavy lon Center

Ping Li

Shaghai Pronton and Heavy lon Center

Zheng Wang

Shanghai Proton and Heavy lon Center

Xin Cai

Shanghai Proton and Heavy lon Center

Guoliang Jiang

Shanghai Proton and Heavy Ion Center

Qing Zhang

Shanghai Proton and Heavy lon Center

\section{Research Article}

Keywords: uterine cervical carcinoma; proton and carbon ion radiotherapy, induction chemotherapy, concurrent chemotherapy

Posted Date: February 4th, 2022

DOI: https://doi.org/10.21203/rs.3.rs-1321735/v1

License: (c) (1) This work is licensed under a Creative Commons Attribution 4.0 International License. Read Full License 


\section{Abstract}

Background: To skip brachytherapy from external radiation and brachytherapy for cervical carcinomas, a combined proton and carbon ion radiotherapy (PCRT) was used. This retrospective study reported the toxicity and short-term outcome.

Methods: 16 cervical SCC patients were consecutively treated with PCRT in our center from August 2016 to July 2019. The acute and late toxicities, overall survival (OS), local control rate (LC), progression-free survival (PFS) and distant metastasis-free survival (DMFS) were analyzed.

Results: For 16 cervical SCC, the prescribed dose for tumor was 76 Gy to 85.6 Gy (relative biologic efficacy (RBE)). With a median follow-up of 34.3 (range, 20.7-56.8) months, all of the acute toxicity were grade $1-2$ with $12.5 \%$ of hepatic toxicity, $75 \%$ of hematological toxicity and $18.8 \%$ of gastroenteric toxicity. No acute urinary toxicity occurred. No severe late toxicity was observed during the follow-up period. At the last follow-up visit, 11 patients were alive, 4 patients died and 1 lost for follow-up; 3 patients had local recurrences and 6 patients had distant metastases. OS, LC, PFS, and DMFS at 1-year, 2-year and 3-year were $100 \%, 100 \%$ and $77.9 \% ; 86.7 \%, 86.7 \%$ and $78.0 \% ; 75.0 \%, 68.8 \%$ and $61.9 \%$; and $87.5 \%, 81.3 \%$ and $75.0 \%$, respectively.

Conclusions: PCRT was feasible to deliver high dose to cervical SCC, and the outcome was quite good. We thought that brachytherapy had the potential to be skipped when PCRT was used and long term results need to be further investigated.

Trial registration: This was a retrospective analysis and it was retrospectively registered. The registration number was NCT05141825 and the date of registration was November $30^{\text {th }}, 2021$.

\section{Background}

Uterine cervical carcinoma is one of the most common cancers of women in the world [1]. Radiotherapy has been widely used to treat uterine cervical carcinoma. For patients with FIGO IB2-IVA radiotherapy combined with cisplatin-based chemotherapy is the standard of care [2]. In terms of radiation technique, the external irradiation, followed by brachytherapy, intracavitary, or interstitial implantation to cervical lesions is commonly used. However, the local control is not satisfactory enough, especially for the bulky tumors. The associated anemia and hypoxia, which are very often in uterine cervical carcinomas, lead tumors to the radio-resistant. Nevertheless, delivery of high dose is limited due to the intolerance of adjacent intestine and colon. Although, brachytherapy could boost dose to the primary tumor, but might not deliver sufficient dose to bulky tumors [3-4]. To improve local control, new radiation technique, proton and heavy ion, had been tried to treat cervical carcinomas.

Because of proton physical dosimetric advantage, it has been used to irradiate uterine cervical carcinoma, and showed the decreased toxicity [5-8]. The most advanced technique, intensity modulated proton therapy (IMPT) with pencil beam scanning could further decrease the doses to small bowel, colon, 
bladder, rectum, head of femur and pelvis [5]. Therefore, it has more potential to spare normal organs, and meanwhile to deliver higher doses to targets compared to photon irradiation [7].

Carbon ion radiotherapy (CIRT) also has the physical dosimetric advantages. Besides, it possess biological advantages due to the nature of high linear energy transfer (LET) in the Bragg peak, which has strong inactivation effect on tumors with relative biological effect (RBE) of 2-3 [9-10], and low oxygen enhancement ratio (OER). National Institute of Radiological Sciences (NIRS), in Japan has completed several phase I/II or phase II clinical trails and showed high dose CIRT could improve the local control of the uterine cervical carcinoma [11]. CIRT with concurrent chemotherapy was safe for bulky tumors and could improve local control and overall survival [12].

Brachytherapy is a necessary part of irradiation for cervical carcinomas. However, we intended to skip it by combined proton and carbon ion radiotherapy (PCRT). Because there are radiation sensitive organs, intestine and colon adjacent to uterine cervical carcinoma, CIRT only would probably injure them more severe and result in more toxicity than proton. Therefore, we used proton for the primary tumor and pelvic nodes and CIRT boost for the clinical cervical tumors and metastatic nodes. In this report, we retrospectively analyzed the cervical carcinoma patients we treated with PCRT to observe its safety, tolerance and local control and survival. This study was approved by Ethical Committee of our center.

\section{Methods}

\section{Patient eligibility}

The patients selection eligibility criteria were: (1). histologically proven cervical squamous cell carcinoma (cervical SCC); (2). International Federation of Gynecology and Obstetrics (FIGO) stage (2014) IB2-IIIB, without rectum invasion; or FIGO stage IA-IB1, who refused surgery or were contraindication for surgery due to comorbidity diseases; (3). received PCRT with or without chemotherapy for curative intention.

From our center database 16 cervical SCC patients from August 2016 to July 2019 were eligible for the analysis. Their clinical characteristics were shown in Table 1. The median age was 48.5 years (range: 30 to 74). The median size of maximal diameter of tumor was $5.5 \mathrm{~cm}$ (range: $2.9-9.5$ ) before treatment. The tumor size decreased after induction chemotherapy in 4 patients. The median size of maximal length of tumor was $7.0 \mathrm{~cm}(4.7-9.5 \mathrm{~cm})$ in those 4 patients and it decreased to $4.8 \mathrm{~cm}(3.2-6.0 \mathrm{~cm})$ after induction chemotherapy. 
Table 1

The clinical characteristics in 16 cervical squamous cell carcinomas

\begin{tabular}{|lc|}
\hline Characteristics & Patient number \\
\hline Age & $48.5(30-74)$ \\
\hline Median (range) (year) & \\
\hline Clinical stage (FIGO) & 2 \\
\hline IB1-B2 & 7 \\
\hline IIA-B & 7 \\
\hline IIIA-B & \\
\hline ECOG & 15 \\
\hline 0 & 1 \\
\hline 1 & \\
\hline Anemia before radiotherapy & 7 \\
\hline Yes & 9 \\
\hline No & \\
\hline Induction chemotherapy & 7 \\
\hline Yes & 9 \\
\hline No & \\
\hline Concurrent chemotherapy & \\
\hline Yes & \\
\hline No & \\
\hline$*$ One patient only received 1 cycle of current chemotherapy \\
\hline
\end{tabular}

\section{PCRT}

Patients were requested to keep the same cookbook and avoid aerogenous food during irradiation period in order to decrease the gas in intestine and colon. Before CT simulation and each irradiation fraction patients were requested to have similar bladder urine filling around 150-200 $\mathrm{ml}$ by keeping the same drinking habit, and rectum should be kept empty by enema. Patients were immobilized in the supine position on the individualized vacuum lock with thermoplastic mask on patients' pelvic. Two sets of 3mm-thick CT were acquired for treatment planning: plain scan and enhancement scan. Pelvic MRI was acquired when needed. 
Gross tumor volume (GTV) was defined as the primary lesion in cervix identified by MRI or PET. Clinical target volume (CTV) consisted of CTV1, CTV2, CTV3 and CTVIn. CTV1 was defined as the pelvic lymph node regions (common iliac, internal iliac, external iliac, obrurator and presacral node regions) and suspicious lymph nodes (LN). CTV2 was defined as high-risk clinical region, including CTV3, uterus, cervix not included in GTV, bilateral parametrium, ovaries, subterminal vagina. CTV3 was defined as GTV expanded with a 5-mm margin. CTVln was defined as enlarged lymph nodes with a 5-mm margin. Planning target volume (PTV) included CTV plus a 5-mm margin for positioning uncertainty, and more margins at the beam directions.

The prescribed doses were listed in Table 2. Proton of 46 Gy (RBE) in 23 fractions was delivered to CTV1. Various proton plus carbon or pure carbon ion boost doses were given to GTV or CTVIn. Biological effect dose (BED) was calculated by Linea-Quadratic model with $\alpha / \beta$ of $10 \mathrm{~Gy}$.

PCRT quality assurance included water phantom dose verification before irradiation; KV X-ray position verification films before each irradiation; and CT review weekly and necessary plan modification when needed.

Table 2

The list of PIRT doses in 16 cervical SCC.

\begin{tabular}{|llll|}
\hline Does & $\begin{array}{l}\text { Total dose (Gy } \\
\text { (RBE) }\end{array}$ & $\begin{array}{l}\text { BED10 } \\
\text { (Gy) }\end{array}$ & $\begin{array}{c}\text { Patient } \\
\text { number }\end{array}$ \\
\hline $\begin{array}{l}\text { proton 46Gy (RBE)/23fx with carbon ion boost of } \\
\text { 30Gy (RBE)/10fx* }\end{array}$ & 76 & 94.2 & 4 \\
\hline $\begin{array}{l}\text { proton 46Gy (RBE)/23fx with carbon ion boost of } \\
\text { 35Gy (RBE)/10fx }\end{array}$ & 81 & 102.7 & 2 \\
\hline $\begin{array}{l}\text { proton 46Gy (RBE)/23fx plus carbon 35.2Gy } \\
\text { (RBE)/8fx }\end{array}$ & 81.2 & 105.9 & 1 \\
\hline $\begin{array}{l}\text { proton 46Gy (RBE)/23fx with carbon ion boost of } \\
\text { 36Gy (RBE)/9fx }\end{array}$ & 82 & 105.6 & 7 \\
\hline $\begin{array}{l}\text { proton 46Gy (RBE)/23fx with carbon ion boost of } \\
\text { 39.6Gy (RBE)/9fx }\end{array}$ & 85.6 & 112.2 & 1 \\
\hline $\begin{array}{l}\text { proton 62Gy (RBE)/31fx with carbon ion boost of } \\
\text { 15Gy (RBE)/5fx }\end{array}$ & 77 & 93.9 & 1 \\
\hline * 46GyE (RBE) to CTV1; various carbon ion doses to GTV or CTVln. & & \\
\hline
\end{tabular}

\section{Chemotherapy}

Seven patients received induction chemotherapy for 1 to 4 cycles before PCRT. During PCRT 13 patients received concurrent chemotherapy with cisplatin $\left(40 \mathrm{mg} / \mathrm{m}^{2}\right.$, once a week) for 6 to $7 \mathrm{cycles}$, but 1 patient, for 1 cycle.

\section{Follow-up and statistics}


Acute toxicity (occurring within 90 days from the start of radiation) was evaluated by National Cancer Institute Common Terminology Criteria for Adverse Events (CTCAE) version 4.03, and the late toxicity (continuing or occurring beyond 90 days), by Radiation Therapy Oncology Group (RTOG). Patients were examined weekly during radiation period and followed-up monthly for 3 months, and every 3 months afterwards. Local failure and distant metastasis were diagnosed based on clinical examinations and/or the evidence of $C T, M R I, P E T$, or biopsy. Overall survival (OS), local control rate (LC), progression-free survival (PFS) and distant metastasis-free survival (DMFS) were estimated from the first day of PCRT by Kaplan-Meier method. Cox's proportional hazard model was used for multivariate analysis. All statistical analyses were performed by SPSS Statistics (SPSS Inc., Chicago, IL, USA).

\section{Results}

\section{Tolerance and toxicity}

All of 16 patients tolerated PCRT very well, and completed PCRT as the planned. None of the patients had their PCRT interrupted because of the toxicity. The last follow-up was performed in September 2021 with the median follow-up time of 34.3 months (range, 20.7-56.8).

All of the acute toxicity were grade $1-2$ with $12.5 \%$ of hepatic toxicity, $75 \%$ of hematological toxicity and $18.8 \%$ of gastroenteric toxicity. No acute urinary toxicity occurred. No severe late toxicity was observed during the follow-up period.

\section{Tumor control and survival}

At the last follow-up visit, 11 patients were alive; 1 patients died of local failure; 3 patients died of distant metastasis; and 1 patient got lost for follow-up. Overall, 3 patients had local recurrences and 6 patients had distant metastases. OS, LC, PFS, and DMFS at 1-year, 2-year and 3-year were 100\%, 100\% and 77.9\%; $86.7 \%, 86.7 \%$ and $78.0 \% ; 75.0 \%, 68.8 \%$ and $61.9 \%$; and $87.5 \%, 81.3 \%$ and $75.0 \%$, respectively (Figure 1 ).

\section{Prognostic factors}

From univariate analysis (Table 3-5) stage corrected with OS $(p=0.025)$ (3-year OS of $100 \%$ in stage IB1IIB2 vs. $40.0 \%$ in stage IIIA-IIIB). The tumor size before any treatment predicted OS $(p=0.037)$ (3-year OS of $100 \%$ in the tumors with maximal GTV length of $<=5.5 \mathrm{~cm}$ vs. $57.1 \%$ in those $>5 \mathrm{~cm}$ ). Other clinical parameters did not show any prognostic values. Multivariate analysis showed that neither of those factors were independent favorable predictors for OS. 
Table 3

Univariate analysis for overall survival (OS) in 16 cervical SCC patients.

\begin{tabular}{|lllll|}
\hline Variable & subgroup & $\mathbf{n}$ & 3-year OS (\%) & p value \\
\hline Concurrent chemotherapy & No & 3 & 66.7 & 0.663 \\
\cline { 2 - 5 } & Yes & 13 & 80.8 & \\
\hline Induction chemotherapy & No & 9 & 77.8 & 0.478 \\
\cline { 2 - 5 } & Yes & 7 & 80.0 & \\
\hline Stage & IB1-IIB2 & 9 & 100.0 & 0.025 \\
\cline { 2 - 5 } & IIIA-IIIB & 7 & 40.0 & \\
\hline Regional node metastasis & No & 7 & 100.0 & 0.222 \\
\cline { 2 - 5 } & Yes & 9 & 57.1 & \\
\hline Maximal length of GTV before any treatment (cm) & $<=5.5$ & 8 & 100 & 0.037 \\
\cline { 2 - 5 } & $>5.5$ & 8 & 57.1 & \\
\hline Anemia before radiotherapy & No & 9 & 100 & 0.074 \\
\cline { 2 - 5 } & Yes & 7 & 44.4 & \\
\cline { 2 - 5 } & & & \\
\hline
\end{tabular}

Table 4

Univariate analysis of local control (LC) in 16 cervical SCC patients.

\begin{tabular}{|lllll|}
\hline Factor & subgroup & $\mathbf{n}$ & 3 -year LC rate (\%) & P value \\
\hline Concurrent chemotherapy & No & 3 & 66.7 & 0.492 \\
\cline { 2 - 5 } & Yes & 13 & 80.2 & \\
\hline Induction chemotherapy & No & 9 & 87.5 & 0.461 \\
\cline { 2 - 5 } & Yes & 7 & 64.3 & \\
\hline Stage & IB1-IIB2 & 9 & 87.5 & 0.263 \\
\hline IIIA-IIIB & 7 & 71.4 & 0.615 \\
\hline Regional LN metastasis & No & 7 & 83.3 & \\
\cline { 2 - 5 } & Yes & 9 & 77.8 & 0.947 \\
\hline Maximal length of GTV before any treatment $(\mathrm{cm})$ & $<=5.5$ & 8 & 83.3 & \\
\cline { 2 - 5 } & $>5.5$ & 8 & 75.0 & 0.086 \\
\hline Anemia before radiotherapy & No & 9 & 87.5 & \\
\cline { 2 - 5 } & Yes & 7 & 57.1 & \\
\cline { 2 - 5 } & & &
\end{tabular}


Table 5

Univariate analysis of distant metastasis-free survival (DMFS) in 16 cervical SCC patients.

\begin{tabular}{|lllll|}
\hline Factor & subgroup & $\mathbf{n}$ & $\begin{array}{l}\text { 3-year DMFS rate } \\
(\%)\end{array}$ & $\begin{array}{l}\text { P } \\
\text { value }\end{array}$ \\
\hline Concurrent chemotherapy & No & 3 & 100.0 & 0.234 \\
\cline { 2 - 5 } Induction chemotherapy & Yes & 13 & 69.2 & \\
\hline Stage & No & 9 & 88.9 & 0.728 \\
\cline { 2 - 5 } & Yes & 7 & 57.1 & 0.313 \\
\hline Regional LN metastasis & IB1-IIB2 & 9 & 88.9 & \\
\cline { 2 - 5 } & IIIA-IIIB & 7 & 57.1 & 0.959 \\
\hline $\begin{array}{l}\text { Maximal length of GTV before any treatment } \\
\text { (cm) }\end{array}$ & No & 7 & 85.7 & 0.069 \\
\cline { 2 - 5 } & Yes & 9 & 66.7 & \\
\hline Anemia before radiotherapy & $<=5.5$ & 8 & 100.0 & 0.622 \\
\hline & $>5.5$ & 8 & 50.0 & \\
\hline
\end{tabular}

\section{Discussion}

Cervical carcinoma in late stages were believed the radio-resistant due to the tumor hypoxia, which resulted from the associated anemia. Therefore, to achieve good outcome, high dose of radiotherapy is necessary. The external irradiation followed by brachytherapy irradiation, intracavitary or interstitial implantation, has been the standard irradiation technique. Typically, patients were irradiated with external beam radiotherapy of 45 to $50 \mathrm{~Gy}$, and followed by intracavitary brachytherapy with 3 to 5 fractions of 5 to 7 Gy per fraction (BED10 of 72.6Gy-119.5 Gy). However, intracavitary brachytherapy might not deliver a sufficient dose to extensive and bulky tumors in stage III or IVA disease [13-14]. In that case, interstitial brachytherapy could be used [15-16]. However, it was invasive and had limited indications. In an effort to exclude brachytherapy, the dosimetric comparison studies had been done between external irradiation plus brachytherapy and external irradiation alone with stereotactic radiotherapy or IMRT [17-18]. However, the high dose to central tumors by brachytherapy could not be reproduced by stereotactic radiotherapy or IMRT. Moreover, a retrospective analysis showed that curative external irradiation and brachytherapy achieved a higher 5-year cancer specific survival than external irradiation alone in cervical carcinoma, which implied the important role of brachytherapy [19]. However, intracavitary or interstitial implantation brachytherapy brought patients inconvenience, or procedures was invasive. Therefore, most advanced irradiation techniques were tried to deliver intensive doses in a hope to skip the brachytherapy. 
Although our analysis was a retrospective study, we had some basic considerations to implement PCRT for cervical SCC. Our intention was to exclude brachytherapy from the combination of external beam and brachytherapy, and meanwhile to keep a similar outcome as that in the external and brachytherapy. From dosimetric comparative study of cervical carcinoma, intensity-modulated proton radiotherapy (IMPT) had a significant reduction for the mean dose of small bowel and functional bone marrow than photon techniques such as IMRT, helical tomotherapy and RapidArc [5-6]. Furthermore, mean dose to the bladder and rectum was decreased by 7-9 Gy with IMPT in patents with pelvic radiation [5]. Volume of pelvic bone marrow receiving $10 \mathrm{~Gy}-20 \mathrm{~Gy}$ or $40 \mathrm{~Gy}$ was associated with hematologic toxicity [20-24]. Thus, protons offered the best sparing of small bowel, rectum and pelvic bone marrow, and could contribute to a significant reduction of acute and late gastrointestinal and hematologic toxicity in cervical carcinoma radiotherapy, especially when combined with concurrent chemotherapy. However, on the other hand, cervical carcinoma often contained a large percentage of radio-resistant hypoxic cells due to the associated anemia. The proton, as low LET beam, would probably not be effective enough to sterilize hypoxic cells. Carbon ion beam is a high LET beam and possesses both physical and biological advantages. From experimental and clinical studies, it had shown strong capabilities to inactivate malignancies with RBE of 2 to 3 . Moreover, it could overcome tumor hypoxia with decreased OER of around 2. Actually, CIRT had been used to treat cervical carcinomas and yielded good outcome for locally advanced bulky cervical SCC [11]. Therefore, we irradiated the cervical tumor and pelvic nodes by proton of 46Gy (RBE), and followed by CIRT boost doses to cervical gross tumor and the metastatic nodes.

From 16 consecutively treated patients in our center by PCRT with 76 Gy (RBE) -85.6 Gy (RBE) (BED10 of 93.9Gy-112.2Gy), LC and OS rates at 1-year, 2-year and 3-year were $86.7 \%, 86.7 \%$ and $78.0 \% ; 100 \%, 100 \%$ and $77.9 \%$ respectively. This result was as good as that reported by modern external irradiation and brachytherapy (Table 6) [3-4, 25-30]. Also it was comparable to NIRS reports [29, 30]. All acute toxicity in our patients were Grade 1-2, and no severe late toxicity occurred during the follow-up period. Compared with that in external beam radiotherapy plus brachytherapy, or CIRT, the toxicity of PCRT seemed to be less [3, 25-28, 30-31].

As proton and CIRT are new radiation techniques for cervical carcinomas, some technical problems we have to deal with, including uterine motion [32], and position variations of bladder and rectum [33]. The above problems would result in dose delivery uncertainty. We applied some measurements to decrease the gas in intestine and colon, to keep the same bladder urine volume and rectum empty. Recently we took CT by in-room CT just before the dose delivery and confirmed the effectiveness of those measurements on keeping anatomy position consistent.

Concurrent platinum-based chemotherapy is a part of the current standard treatment for patients with FIGO stage IB2-IVA. NIRS carried out a dose-escalation study on locally advanced cervical SCC by CIRT alone in 2014, and showed 2-year OS and LC were $64 \%$ and $68.2 \%$, respectively [11]. In 2019, NIRS did another phase $1 / 2$ study on CIRT, but with concurrent chemotherapy, and the 2-year OS and LC were $82 \%$ and $67 \%$, respectively [31]. The 2-year OS was improved from $64-82 \%$ in concurrent chemo-radiation study, which demonstrated again the importance of concurrent chemotherapy in CIRT as the same as in 
photon irradiation. However, concurrent chemotherapy did not improve the outcome in our study probably due to the small example size.

Univariate analysis in our study showed that only stage and tumor size were related to OS as that reported in literature [31]. If we could make the disease down staged before irradiation, it would be of help. Induction chemotherapy might be a strategy to reduce tumor burden in locally advanced cervical carcinomas. A British phase II trail included 46 patients with FIGO stage Ib2-IVa cervical carcinoma showed a good response rate to induction chemotherapy before CCRT, and the 5-year OS was 67\% [34], which was better than the outcome after CCRT alone [35]. An international randomized multicenter phase III trial, INTERLACE (NCT01566240) is going on to compare the induction chemotherapy followed by CCRT and CCRT alone. In our center, we are planning to initiate a prospective clinical trial of induction chemotherapy, and concurrent chemotherapy and PCRT for locally advanced cervical carcinomas to further confirm PCRT feasibility and its efficacy.

\section{Conclusions}

In summary, our preliminary result demonstrated that PCRT was feasible to deliver high dose to cervical SCC, and the outcome was quite good. We thought that brachytherapy had the potential to be skipped when PCRT was used. However, as it was a retrospective analysis with small sample size and short follow-up period, the conclusion was very preliminary and long term results need to be further investigated.

Table 6. Comparison of clinical outcomes for cervical carcinomas

\section{Abbreviations}

PCRT: Proton and carbon ion radiotherapy; OS: Overall survival; LC: Local control rate; PFS: Progressionfree survival; DMFS: Distant metastasis-free survival; OAR: Organ at risk; NIRS: National Institute of Radiological Sciences of Japan; CIRT: Carbon ion radiotherapy; SPHIC: Shanghai Proton and Heavy lon Center; SOP: Standard operating procedure; FIGO: International Federation of Gynecology and Obstetrics; CT: Computed tomography; MRI: Magnetic resonance imaging; PET: Positron emission tomography; ECOG: Eastern Cooperative Oncology Group; GTV: Gross tumor volume; CTV: Clinical target volume; PTV: Planning targer volume; LN: Lymph nodes; SIB: Simultaneously integrated boost; BED: Biologically effective dose; CTCAE: Common Terminology Criteria for Adverse Events; RTOG: Radiation Therapy Oncology Group; RT = radiotherapy; EBRT = external beam radiotherapy; IGBT = image-guided brachytherapy;

\section{Declarations}

Ethics approval and consent to participate 
The study was approved by Ethics Committee of Shanghai Proton and Heavy lon Center.

Consent for publication

Not applicable

Availability of data and materials

The data and materials of this study are available from the corresponding author on reasonable request.

Competing interests

The authors declare that they have no competing interests

Funding

No funding

Authors' contributions

QZ is responsible for editorial correspondence and have contributed to all of the following: (1) the conception and design of the study, and acquisition, analysis and interpretation of data, (2) contributing reagents/materials/analysis tools, (3) revising the article, (4) final approval of the version to be submitted. ZY, ZSH, PL, ZW, XC, and GLJ have contributed to as the following: (1) acquisition, analysis and interpretation of data, (2) drafting and revising the article. ZY and ZSH were major contributors in writing the manuscript. All authors read and approved the final manuscript.

Acknowledgements

We acknowledge all the investigators, patients and their families.

Authors' information

Qing Zhang, Email: qing.zhang@sphic.org.cn

Footnotes

Zhan Yu and Zhengshan Hong contributed equally to this work.

\section{References}

1. Arbyn M, Weiderpass E, Bruni L, de Sanjosé S, Saraiya M, Ferlay J, et al. Estimates of incidence and mortality of cervical carcinoma in 2018: a worldwide analysis. Lancet Glob Health. 2020 Feb;8(2):e191-203. 
2. NCCN Guideline Cervical carcinoma. Version 1, 2022, NCCN Clinical Practice Guideline in Oncology. https://www.nccn.org.

3. Kusada T, Toita T, Ariga T, Maemoto H, Hashimoto S, Shiina H, et al. Computed tomography-based image-guided brachytherapy for cervical carcinoma: Correlations be- tween dose-volume parameters and clinical outcomes. J Radiat Res. 2018;59:67-76.

4. Minkoff D, Gill BS, Kang J, Beriwal S. Cervical carcinoma outcome prediction to high-dose rate brachytherapy using quantitative magnetic resonance imaging analysis of tumor response to external beam radiotherapy. Radiother Oncol. 2015;115:78-83.

5. Marnitz S, Wlodarczyk W, Neumann O, Koehler C, Weihrauch M, Budach V, et al. Which technique for radiation is most beneficial for patients with locally advanced cervical carcinoma? Intensity modulated proton therapy versus intensity modulated photon treatment, helical tomotherapy and volumetric arc therapy for primary radiation - an intraindividual comparison. Radiat Oncol. 2015;10:91.

6. Dinges E, Felderman N, McGuire S, Gross B, Bhatia S, Mott S, et al. Bone marrow sparing in intensity modulated proton therapy for cervical carcinoma: Efficacy and robustness under range and setup uncertainties. Radiother Oncol. 2015;115(3):373-8.

7. Hashimoto S, Shibamoto Y, Iwata H, Ogino H, Shibata H, Toshito T, et al. Whole-pelvic radiotherapy with spot-scanning proton beams for uterine cervical carcinoma: a planning study. J Radiat Res. 2016;57:524-32.

8. de Boer P, van de Schoot AJAJ, Westerveld H, Smit M, Buist MR, Bel A, et al. Target tailoring and proton beam therapy to reduce small bowel dose in cervical carcinoma radiotherapy: A comparison of benefits. Strahlenther Onkol. 2018;194:255-63.

9. Kanai T, Furusawa Y, Fukutsu K, Itsukaichi H, Eguchi-Kasai K, Ohara H. Irradiation of mixed beam and design of spread-out Bragg peak for heavy-ion radiotherapy. Radiat Res. 1997;147:78-85.

10. Kanai T, Endo M, Minohara S, Miyahara N, Koyama-ito H, Tomura H, et al. Biophysical characteristics of HIMAC clinical irradiation system for heavy-ion radiation therapy. Int J Radiat Oncol Biol Phys. 1999;44:201-10.

11. Wakatsuki M, Kato S, Ohno T, Karasawa K, Ando K, Kiyohara H, et al. Dose-escalation study of carbon ion radiotherapy for locally advanced squamous cell carcinoma of the uterine cervix (9902). Gyne Oncol. 2014;132(1):87-92.

12. Okonogi N, Wakatsuki M, Kato S, Karasawa K, Kiyohara H, Shiba S, et al. Clinical outcomes of carbon ion radiotherapy with concurrent chemotherapy for locally advanced uterine cervical adenocarcinoma in a phase 1/2 clinical trial (Protocol 1001). Cancer Med. 2018;7(2):351-9.

13. Nakano T, Kato S, Ohno T, Tsujii H, Sato S, Fukuhisa $K$, et al. Long-term results of high-dose-rate intracavitary brachytherapy for squamous cell carcinoma of the uterine cervix. Cancer. 2005;103(1):92-101.

14. Parker K, Gallop-Evans E, Hanna L, Adams M. Five years' experience treating locally advanced cervical carcinoma with concurrent chemoradiotherapy and high-dose-rate brachytherapy: results 
from a single institution. Int J Radiat Oncol Biol Phys. 2009;74(1):140-6.

15. Syed AM, Puthawala AA, Abdelaziz NN, el-Naggar M, Disaia P, Berman M, et al. Long-term results of low-dose-rate interstitial-intracavitary brachytherapy in the treatment of carcinoma of the cervix. Int $J$ Radiat Oncol Biol Phys. 2002;54(1):67-78.

16. Wakatsuki M, Ohno T, Yoshida D, Noda SE, Saitoh J, Shibuya K, et al. Intracavitary combined with CTguided interstitial brachytherapy for locally advanced uterine cervical carcinoma: introduction of the technique and a case presentation. J Radiat Res. 2011;52(1):54-8.

17. Georg D, Kirisits C, Hillbrand M, Dimopoulos J, Pötter R. Image-guided radiotherapy for cervix cancer: high-tech external beam therapy versus high-tech brachytherapy. Int J Radiat Oncol Biol Phys. 2008;71(4):1272-8.

18. Otahal B, Dolezel M, Cvek J, Simetka O, Klat J, Knybel L, et al. Dosimetric comparison of MRI- based HDR brachytherapy and stereotactic radiotherapy in patients with advanced cervical carcinoma: a virtual brachytherapy study. Rep Pract Oncol Radiother. 2014;19(6):399-404.

19. Kim YA, Yang MS, Park M, Choi MG, Kim SY, Kim YJ. Brachytherapy utilization rate and effect on survival in cervical carcinoma patients in Korea. J Gynecol Oncol. 2021;32(6):e85.

20. Albuquerque K, Giangreco D, Morrison C, Siddiqui M, Sinacore J, Potkul R, et al. Radiation-Related Predictors of Hematologic Toxicity After Concurrent Chemoradiation for Cervical carcinoma and Implications for Bone Marrow-Sparing Pelvic IMRT. Int J Radiat Oncol Biol Phys. 2011;79:1043-7.

21. Mell LK, Schomas DA, Salama JK, Devisetty K, Aydogan B, Miller RC, et al. Association between bone marrow dosimetric parameters and acute hematologic toxicity in anal cancer patients treated with concurrent chemotherapy and intensity-modulated radiotherapy. Int J Radiat Oncol Biol Phys. 2008;70:1431-7.

22. Platta CS, Bayliss A, McHaffie D, Tome WA, Straub MR, Bradley KA. A dosimetric analysis of tomotherapy based intensity modulated radiation therapy with and without bone marrow sparing in gynecologic malignancies. Technol Cancer Res T. 2013;12:19-29.

23. Rose BS, Aydogan B, Liang Y, Yeginer M, Hasselle MD, Dandekar V, et al. Normal tissue complication probability modeling of acute hematologic toxicity in cervical carcinoma patients treated with chemoradiotherapy. Int J Radiat Oncol Biol Phys. 2011;79:800-7.

24. Klopp AH, Moughan J, Portelance L, Miller BE, Salehpour MR, Hildebrandt E, et al. Hematologic toxicity in RTOG 0418: a phase 2 study of postoperative IMRT for gynecologic cancer. Int J Radiat Oncol Biol Phys. 2013;86:83-90.

25. Chen CC, Lin JC, Jan JS, Ho SC, Wang L. Definitive intensity-modulated radiation therapy with concurrent chemotherapy for patients with locally advanced cervical carcinoma. Gynecol Oncol. 2011;122(1):9-13.

26. Pötter R, Georg P, Dimopoulos JC, Grimm M, Berger D, Nesvacil N, et al. Clinical outcome of protocol based image (MRI) guided adaptive brachytherapy combined with 3D conformal radiotherapy with or without chemotherapy in patients with locally advanced cervical carcinoma. Radiother Oncol. 2011;100:116-23. 
27. Sturdza A, Po"tter R, Fokdal LU, Haie-Meder C, Tan LT, Mazeron R, et al. Image guided brachytherapy in locally advanced cervical carcinoma: Improved pelvic control and survival in RetroEMBRACE, a multicenter cohort study. Radiother Oncol. 2016;120:428-33.

28. Tiwari R, Narayanan GS, Narayanan S, Suresh Kumar P. Long-term effectiveness and safety of image-based, transperineal combined intracavitary and interstitial brachytherapy in treatment of locally advanced cervical carcinoma. Brachytherapy. 2020;19(1):73-80.

29. Zhang N, Tang Y, Guo X, Mao Z, Yang W, Cheng G. Analysis of dose-effect relationship between DVH parameters and clinical prognosis of definitive radio(chemo)therapy combined with intracavitary/interstitial brachytherapy in patients with locally advanced cervical carcinoma: A singlecenter retrospective study. Brachytherapy. 2020;19(2):194-200.

30. Okonogi N, Wakatsuki M, Kato S, Shiba S, Kobayashi D, Kiyohara H, et al. Long-term outcomes of carbon-ion radiotherapy for locally advanced squamous cell carcinoma of the uterine cervix. Anticancer Res. 2018;38(1):457-63.

31. Okonogi N, Wakatsuki M, Kato S, Karasawa K, Miyasaka Y, Murata H, et al. A Phase 1/2 Study of Carbon Ion Radiation Therapy With Concurrent Chemotherapy for Locally Advanced Uterine Cervical Squamous Cell Carcinoma (Protocol 1302). Int J Radiation Oncol Biol Phys. 2019;104(3):631-9.

32. Gordon JJ, Weiss E, Abayomi OK, Siebers JV, Dogan N. The effect of uterine motion and uterine margins on target and normal tissue doses in intensity modulated radiation therapy of cervical carcinoma. Phys Med Biol. 2011;56(10):2887-901.

33. Jadon R, Pembroke CA, Hanna CL, Palaniappan N, Evans M, Cleves AE, et al. A systematic review of organ motion and image-guided strategies in external beam radiotherapy for cervical carcinoma. Clin Oncol. 2014;26:185-96.

34. McCormack M, Kadalayil L, Hackshaw A, Hall-Craggs MA, Symonds RP, Warwick V, et al. A phase II study of weekly neoadjuvant chemotherapy followed by radical chemoradiation for locally advanced cervical carcinoma. Br J Cancer. 2013;108(12):2464-9.

35. Vale CL, Tierney JF, Davidson SE, Drinkwater KJ, Symonds P. Substantial improvement in UK cervical carcinoma survival with chemoradiotherapy: results of a Royal College of Radiologists' audit. Clin Oncol (R Coll Radiol). 2010;22(7):590-601.

\section{Figures}


A

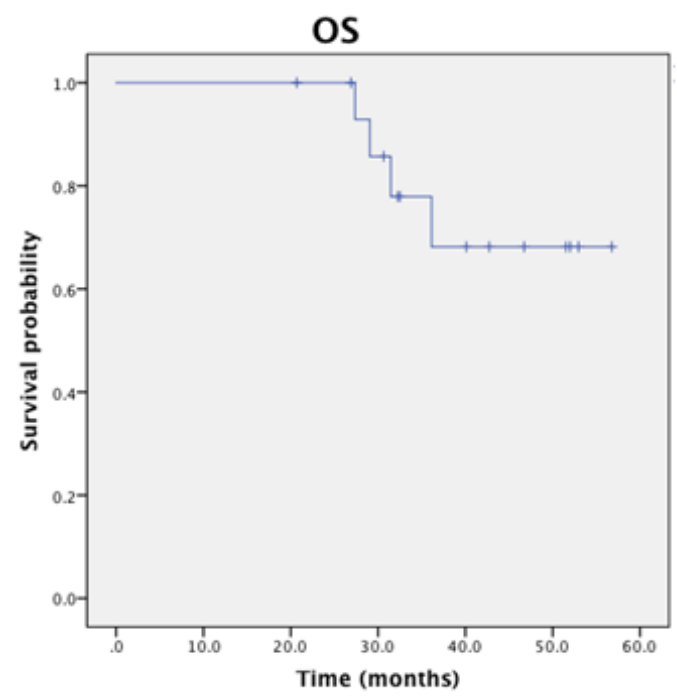

C

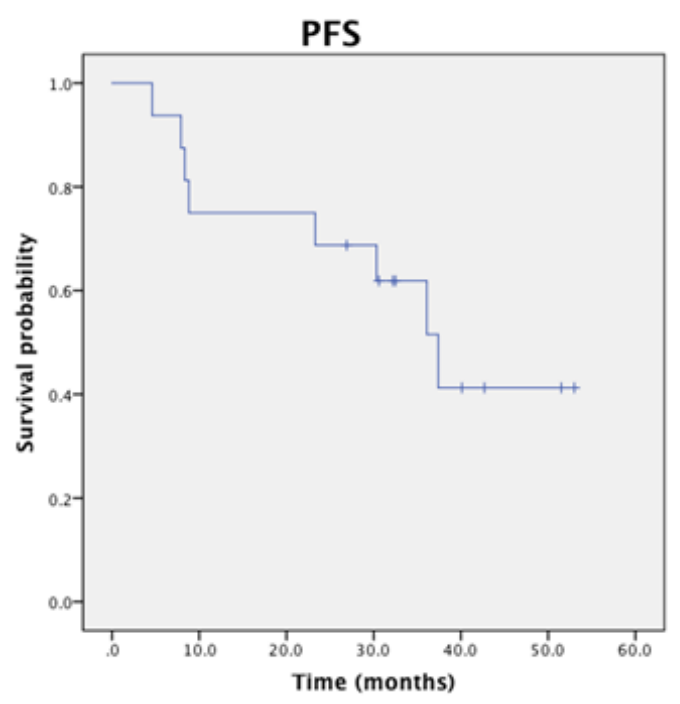

B

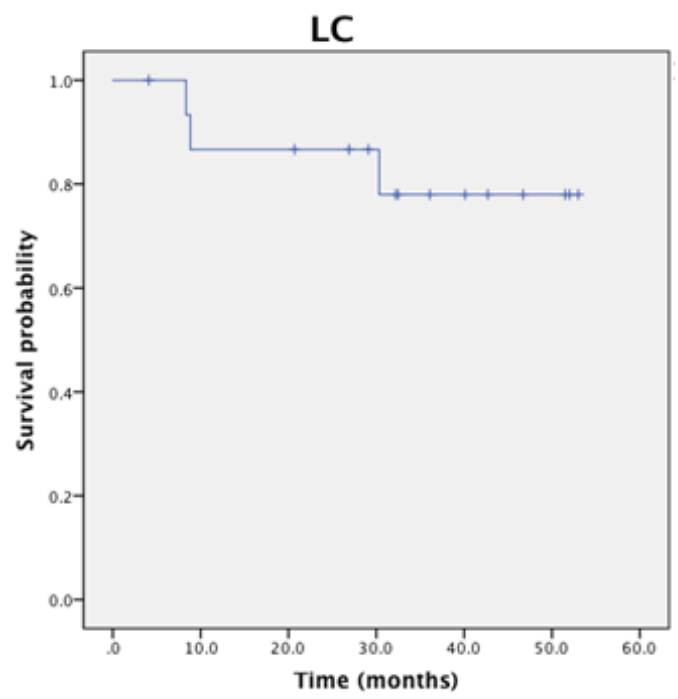

D

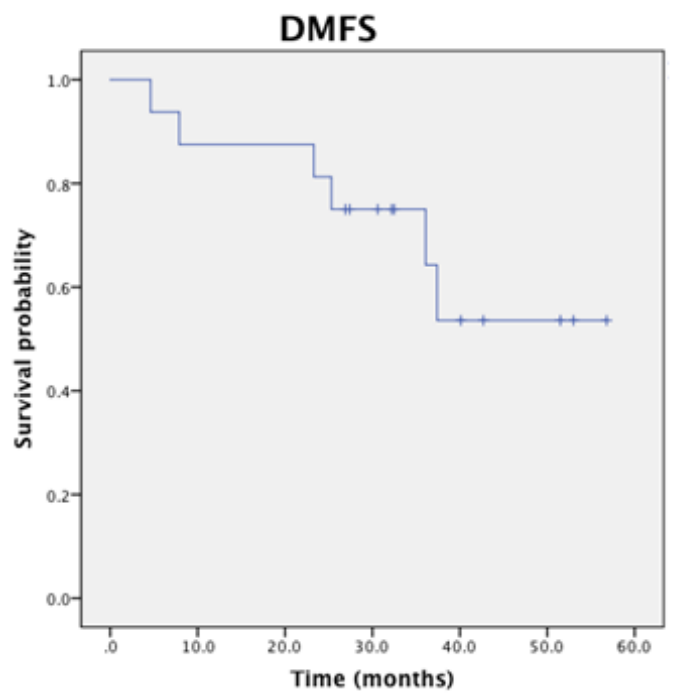

Figure 1

(A) Overall survival (OS), (B) local control (LC), (C) progression-free survival (PFS) and (D) distant metastasis-free survival (DMFS) in 16 cervical SCC. 